\title{
Left ventricular dysfunction by conventional and tissue Doppler echocardiography in pediatric hemodialysis patients: relation with plasma brain natriuretic peptide levels
}

Fatma A. Mostafa ${ }^{1}$, Inas A.E.S. Sad ${ }^{1}$, Manal F. Elshamaa², Ahmed M. Badr ${ }^{1}$, Soha Abd. Eldayem², Ingy Ashmawy ${ }^{3}$, Yomna A.E.M. Abd Elrahim²

${ }^{1}$ Pediatrics Department, Faculty of Medicine, Cairo University, Cairo, Egypt
2Pediatrics Department and Medical Research Centre of Excellence (MRCE), National
Research Centre, Cairo, Egypt
${ }^{3}$ Clinical and Chemical Pathology Department, National Research Centre, Cairo, Egypt

Submitted: 30 November 2017

Accepted: 4 January 2018

Arch Med Sci Atheroscler Dis 2018; 3: e18-e28

DOI: https://doi.org/10.5114/amsad.2018.73277

Copyright (c) 2018 Termedia \& Banach

\section{Abstract}

Introduction: Tissue Doppler imaging (TDI) is a relatively new echocardiography method in children with chronic kidney disease (CKD). The aims of this study were to evaluate left ventricular (LV) diastolic function in pediatric CKD patients using conventional pulsed-wave Doppler echocardiography (CPWD) and TDI methods and correlate them with BNP levels.

Material and methods: Thirty children on regular hemodialysis (HD) were included. Left ventricular systolic and diastolic indices and BNP levels were measured immediately before and after HD.

Results: After HD, LV and left atrium diameters were significantly decreased ( $p<0.001$ and $p<0.001$, respectively). Transmitral E velocity and E/A ratio decreased ( $p<0.001$ and $p<0.001$, respectively). Also, there were significant decreases in the early diastolic velocity E' of LV wall, septal wall, and anterior wall ( $p=0.001, p=0.004$ and $p<0.001$, respectively). Mean $E / E$ ' ratio and ratios of septal and lateral walls were decreased significantly ( $p=0.004, p=0.002$ and $p=0.017$, respectively). BNP levels decreased significantly during HD when comparing concentrations before and after HD $(p<0.001)$. BNP strongly correlated with LV diastolic function indices (before HD: mean E/E' $(r=0.401, p=0.028)$, lateral E/E" $(r=0.291, p=0.025)$, septal E' $(r=-0.398, p=0.029)$, lateral wall E' $(r=-0.452, p=0.012)$ and mean E' $(r=-0.469, p=0.009)$, after HD: mean E/E' $(r=0.38, p=0.038)$ and lateral E/E" $(r=0.474, p=0.008)$ and lateral wall E' $(r=-0.270, p=0.037))$. Conclusions: The CPWD and TDI-derived LV indices are influenced by HD. The diagnostic utility of BNP in the presence of deteriorating renal function may be compromised to some extent.

Key words: hemodialysis, left ventricular diastolic function, conventional Doppler echocardiography, tissue Doppler echocardiography, BNP levels.

\section{Introduction}

Cardiovascular disease (CVD) is considered as the predominant cause of mortality and morbidity in chronic kidney disease (CKD) patients [1]. Left ventricular diastolic and systolic dysfunction and left ventricular hy-

\section{Corresponding author:} Prof. Manal F. Elshamaa MD Pediatrics Department National Research Centre 33 Ebuhous St Dokki, 12311 Cairo, Egypt

Phone: +20 233371010 E-mail: manal_elshmaa@ hotmail.com 
pertrophy (LVH) contribute to the increased cardiovascular mortality rate in these patients [2, 3]. Such changes have been observed in young adults and children on prolonged dialysis [4-6].The cardiovascular mortality and morbidity are seen in earlier stages of CKD, and the risk is increased by multiple risk factors such as sodium and fluid retention, hypertension, anemia, inflammation and hyperparathyroidism [7].

Left ventricular hypertrophy is a common finding in CKD patients [8] and its severity increases with increasing severity of CKD [9]. Initially, LVH is discussed as a physiological response to volume and pressure overload. However, sustained overload in combination with CKD associated risk factors may result in maladaptive LVH characterized by structural changes in the myocardium (calcification, fibrosis and collagen accumulation), resulting in diastolic and systolic dysfunction [10, 11].

Causes of LV diastolic dysfunction are impaired active LV relaxation or decreased LV compliance. These changes are reflected in low diastolic volume for a given diastolic pressure, meaning reduced passive LV filling [1]. The decrease in peak E velocity, with a resultant decrease in the E/A ratio, occurs due to a decrease in ventricular preload leads. This could have an effect on the obtained decrease of E/A ratio after hemodialysis (HD). Further, children with mild hypertension had an increase of peak $A$ velocity probably due to increased LV chamber stiffness caused by LVH [12].

Myocardial function and LVH in CKD patients have been studied using conventional pulsedwave Doppler echocardiography (cPWD) in both pediatric and adult patients on regular HD, and this method evaluates hydrodynamic responses, which are load-dependent, making it disadvantageous in end-stage renal disease (ESRD), where fluid status may change according to the patient's condition [2-6]. Conventional pulsedwave Doppler echocardiography only allows partially subjective semi-quantitative measurements. Furthermore, it has been shown to have low sensitivity to differentiate between pathological and physiological LVH and diastolic dysfunction in patients with LVH [13] and normal ejection fraction (EF) [14].

Tissue Doppler imaging (TDI) as a relatively new echocardiography method is a quantitative and objective technique for evaluating myocardial tissue functions in contrast to conventional echocardiography. Tissue Doppler imaging monitors directly the evoked hydrodynamic responses of the mechanical wall function [15]. Moreover, TDI has been described as less preload dependent with greater sensitivity regarding the parameters of the diastolic and systolic function, compared to those measured by CPWD [16].
Tissue Doppler imaging measurements of regional myocardial velocities allow simultaneous estimations of systolic function (ejection phase and isovolumetric contraction phase), the filling pressure of LV, time intervals, and diastolic function. Studies have demonstrated that systolic velocities are good indicators of LV function, having a good correlation with LV global ejection fraction and invasive measurements of LV contractility [15]. Moreover, diastolic velocities are correlated with the time constant of LV relaxation and inversely with LV pressures and volumes obtained by invasive catheter-tip micromanometry [14].

Tissue Doppler imaging was proved to be a superior evaluation method to CPWD in patients with impaired LV relaxation, regardless of the flow pattern of the mitral inflow velocity (impaired relaxation or pseudonormalization or restriction) $[17,18]$. Some studies have reported that in HD patients, myocardial dysfunction which could not be detected by cPWD can be detected by TDI [19]. But it is not known whether TDI can give additional information on LV function at earlier stages in CKD patients or not.

B-type natriuretic peptide (BNP) is a marker of CVD which is a common complication in adults with CKD, but the range and predictive power of BNP concentrations in children with CKD are still not known [20].

This study aimed to evaluate LV diastolic function in pediatric HD patients, to compare the findings obtained with two modalities, CPWD and TDI, and to study the relationship between BNP level and LV diastolic function in HD children without symptomatic cardiac complications.

\section{Material and methods}

\section{Study design}

This study was designed as a prospective cohort investigation through which echocardiographic parameters were measured and BNP concentrations were assayed within $30 \mathrm{~min}$ pre-HD and 30 min after HD in consecutive ESRD children. This research study was performed between May 2013 and February 2016 in the hemodialysis unit of the Center of Pediatric Nephrology and Transplantation (CPNT), Children's Hospital.

\section{Patient selection}

The study population consisted of 30 patients suffering from ESRD and on regular HD for more than 6 months with an absence of primary myocardial disease. Exclusion criteria included valvular lesions beyond mild severity, atrial fibrillation or paced rhythm, pericardial effusion with evident cardiac compression by echocardiography, constrictive pericarditis, patients with an advanced 
chronic obstructive pulmonary disease, interstitial pneumopathy and chronic thromboembolic disease. Children received standard bicarbonate containing bath dialysis treatment three times per week for 3 to $4.5 \mathrm{~h}$ in each session (with blood flow rate $5-7 \mathrm{ml} / \mathrm{kg}$, and dialyzer surface area $1 \mathrm{~m}^{2}, 7 \mathrm{~m}^{2}$ and $12 \mathrm{~m}^{2}$ polysulfone membrane). Informed consent was obtained from the parents of patients and controls. The study was approved by the Ethics Committees of the National Research Centre and Faculty of Medicine, Cairo University. Hypertension was defined as a diagnosis documentation or by the use of antihypertensive medications.

\section{Measurement of BNP}

\section{Blood sample collection}

Due to the unstable nature of BNP in laboratory samples, special precautions for handling and processing the sample were done: $2 \mathrm{ml} \mathrm{K2}$ ethylene diamine tetra-acetic acid (EDTA) plasma samples were collected through venous puncture from each subject under completely sterile conditions acquired before HD (pre-HD BNP) and after the HD session (post-HD BNP) then transported on ice until they were separated by centrifugation within 30 min of sample collection and immediately stored in aliquots at $-80^{\circ} \mathrm{C}$ for the enzyme-linked immunosorbent assay (ELISA) assay to be done within 3-7 days. Hemolyzed and lipemic samples were excluded. BNP plasma concentration was measured by ELISA according to the manufacturer's instructions (Glory Science Co., Ltd, Cat\# B9050, TX, USA). Echocardiography staff (single observer) did not know the BNP values.

Pre- and post-HD kidney function tests were performed by standard laboratory methods using a Synchron CX7 autoanalyzer (Olympus America Inc., Center Valley, Pennsylvania, USA) and an Olympus AU400 analyzer (Olympus America, Inc., Center Valley, Pa., USA). Estimations of complete blood count $(\mathrm{CBC})$ were made by a Coulter counter, erythrocyte sedimentation rate (ESR) by the Westergen method, serum C-reactive protein (CRP) by ELISA and plasma calcium and phosphorus levels using standard laboratory methods.

\section{Echocardiography}

To minimize the effect of fluid overload, patients on HD had an echocardiographic evaluation before and within 2-3 h after HD treatment, when their weight was close to "dry weight". Conventional echocardiography (M-mode and c-PWD) measurements were performed according to American Society of Echocardiography guidelines 30 min before and after the HD session for all children [21] (and within the same time samples for BNP measurements were obtained) with Vivid 5 Dimension (General Electric Healthcare Company, Milwaukee, WI, China) by use of 3 and $5 \mathrm{MHz}$ combined imaging and Doppler transducers in the left lateral position. Both 2D and Doppler echocardiography were recorded through about five cardiac cycles at 50 or $100 \mathrm{~mm} / \mathrm{s}$ sweep speed.

The cardiac measurements done by $2 D$, involving left ventricular diameter in end diastole (LVEDD), the posterior wall thickness in diastole (PWT), and inter-ventricular septum thickness in end diastole (IVS) were obtained by standard M-mode. Cardiac volumes including left ventricular end-diastolic and end-systolic volumes (LVEDV and LVESV) and LV systolic function were assessed by calculation of ejection fraction (EF) calculated by the Simpson method and complemented by subjective visual estimation and fractional shortening (FS). Ejection fraction $<50 \%$ was used to define systolic dysfunction. Also, left atrial (LA) size and aortic dimension (Ao) were determined.

Profiles of pulsed Doppler transmitral flow velocity were taken with the sample volume positioned from the apical four-chamber view, just below the tip of the mitral leaflet. All the following parameters were measured: peak transmitral flow velocity in early diastole (peak E), peak transmitral flow velocity in late diastole (peak $A$ ), E/A ratio, and $\mathrm{E}$ deceleration time (DT). In addition, isovolumetric relaxation time (IVRT) and R-R interval were measured.

Tissue Doppler imaging was done through the four-chamber view where the mitral annular planes were perpendicular to the ultrasound beam. A $5-\mathrm{mm}$ pulsed TD sample volume was sited at the lateral and the septal aspects of the mitral annulus and also at the lateral aspect of the tricuspid annulus. The global LV systolic and diastolic function were calculated as the average velocities of six LV walls (septal, lateral, inferior, anterior, posterior and anterio-septal). Peak systolic (S') (systolic myocardial function), peak early diastolic (E') (early diastolic filling velocity), and late peak diastolic myocardial (A') (late diastolic filling velocity) velocities, IVRT and Ea/Aa ratio at the lateral and septal mitral and also at the freewall tricuspid annulus were measured. The ratio of $E$ to E' velocity (E/E') was evaluated as a representative of LV filling pressure.

\section{Statistical analysis}

Statistical analysis was performed using SPSS 16 for Windows (SPSS 16, Inc., Chicago, IL, USA). All group data were reported as mean $\pm S D$. To detect normality of variables, the Kolmogorov-Smirnov test was used. Means \pm SDs were used for the 
normally distributed data. The unpaired Student's $t$-test and Mann-Whitney $U$-test were used to assess differences between the patient pre- vs. post-HD findings. Correlation analyses involving blood biochemistry results, 2D M-mode echocardiographic variables, pulsed-Doppler echocardiography measurements and pulsed-DTI results were performed using Pearson's correlation coefficient. Pearson's rank correlation was also performed to evaluate correlations between the plasma of level BNP and all other indices. $P$-values $<0.05$ were considered statistically significant.

\section{Results}

The mean age of the study population at inclusion was $11.4 \pm 2.39$ years. Sixty percent of patients were male. Ten patients had hypertension and were taking antihypertensive medications. Calcium channel blockers (46.6\%), ACE inhibitors $(60 \%)$ and $\beta$-blockers (50\%) were used. The duration of HD treatment was $6.1 \pm 2.87$ years. Clinical and laboratory characteristics of the study population are listed in Table I. The mean measurement of serum phosphorus was $5.86 \pm 1.27 \mathrm{mg} / \mathrm{dl}$, alkaline phosphatase was $713 \pm 590 \mathrm{mg} / \mathrm{dl}$, serum calcium was $8.69 \pm 0.8 \mathrm{mg} / \mathrm{dl}$ and serum urea was $108 \pm 47.64 \mathrm{mg} / \mathrm{dl}$. Two patients were on digoxin and echocardiography was repeated where the echocardiographic measurement had been improved. All dialyzed children were taking vitamin D and oral calcium.
Table I. Demographic and clinical characteristics of patients with end-stage renal disease $(n=30)^{*}$

\begin{tabular}{|c|c|}
\hline Parameter & Frequency \\
\hline Age [years] & $11.4 \pm 2.39(6-14)$ \\
\hline Gender, M/F & $3 / 2(60 \% / 40 \%)$ \\
\hline BMI $\left[\mathrm{kg} / \mathrm{m}^{2}\right]$ & $17.9 \pm 2.8$ \\
\hline Duration of dialysis [years] & $6.1 \pm 2.87(1-13)$ \\
\hline $\mathrm{SBP}[\mathrm{mm} \mathrm{Hg}]$ & $129 \pm 14$ \\
\hline $\mathrm{DBP}[\mathrm{mm} \mathrm{Hg}]$ & $86 \pm 12$ \\
\hline Hemoglobin [mg/dl] & $10.57 \pm 0.98$ \\
\hline Serum calcium [mg/dl] & $8.69 \pm 0.8$ \\
\hline Creatinine $[\mathrm{mg} / \mathrm{dl}]$ & $6.32 \pm 2.26$ \\
\hline Serum phosphorus [mg/dl] & $5.86 \pm 1.27$ \\
\hline Alkaline phosphatase $[\mathrm{mg} / \mathrm{dl}]$ & $713 \pm 590$ \\
\hline Urea [mg/dl] & $108 \pm 47.64$ \\
\hline \multicolumn{2}{|l|}{ Current antihypertensive drugs: } \\
\hline Calcium-channel blocker & $14(46.6 \%)$ \\
\hline ACE inhibitors & $18(60 \%)$ \\
\hline$\beta$-blockers & $15(50 \%)$ \\
\hline
\end{tabular}

${ }^{*}$ Data presented as mean $\pm S D$, percentages or (range) where appropriate. ACE - angiotensin converting enzyme, BMI - body mass index, DBP - diastolic blood pressure, SBP - systolic blood pressure.

Table II. Comparison of M-mode echocardiography findings of the patients before and after HD treatment

\begin{tabular}{|lccc|}
\hline Parameter & Before HD $(n=30)$ & After HD $(n=30)$ & $P$-value \\
\hline HR [beats/min] & $92.8 \pm 15.89$ & $106.5 \pm 17.28$ & $<0.001^{*}$ \\
\hline LVEDD [mm] & $4.21 \pm 0.76$ & $3.90 \pm 0.7$ & $0.001^{*}$ \\
\hline LVEDD, SDS & $0.87 \pm 1.69$ & $0.13 \pm 1.68$ & $0.034^{*}$ \\
\hline LVESD [mm] & $2.66 \pm 0.55$ & $2.54 \pm 0.58$ & 0.5 \\
\hline IVSs [mm] & $1.19 \pm 0.19$ & $1.17 \pm 0.17$ & 0.6 \\
\hline IVSd [mm] & $0.89 \pm 0.21$ & $0.87 \pm 0.17$ & 0.9 \\
\hline IVSd (SDS) & $1.9 \pm 1.07$ & $1.88 \pm 1$ & 0.148 \\
\hline LVPWD [mm] & $0.77 \pm 0.15$ & $0.81 \pm 0.16$ & 0.2 \\
\hline LVPWD, SDS & $1.91 \pm 1.16$ & $2.16 \pm 1.07$ & $<0.001^{*}$ \\
\hline LA dimension [mm] & $3.06 \pm 0.5$ & $2.73 \pm 0.4$ & 0.486 \\
\hline Ao [mm] & $2.15 \pm 0.28$ & $2.11 \pm 0.29$ & 1.0 \\
\hline FS (\%) & $36.8 \pm 5.09$ & $36.8 \pm 6.3$ & 0.699 \\
\hline EF (\%) & $67.1 \pm 6.61$ & $66.73 \pm 7.95$ & \\
\hline
\end{tabular}

Data presented as mean $\pm S D$. Ao - aorta, EF-ejection fraction, FS - fraction shortening, HR - heart rate, IVRT - isovolumetric relaxation time, IVSd - interventricular septum diastole, IVSS - interventricular septum systole, LA - left atrium, LVEDD - left ventricular end diastolic diameter, LVESD - left ventricular end systolic diameter, LVPWD - left ventricular posterior wall diameter, SDS - standard deviation score. ${ }^{\star} P<0.05$ was considered significant. 


\section{Cardiac structure, geometry,} and systolic function

The baseline parameters and the changes of echocardiographic measurements after maintenance HD treatment are presented in Table II. A marked improvement in LVEDD occurred after the HD session (before HD $4.21 \pm 0.76 \mathrm{~mm}$ vs. after HD $3.9 \pm 0.7 \mathrm{~mm}, p<0.001)$ and was confirmed by the standard deviation score (SDS) $(p=0.001)$. Heart rate was significantly higher after the HD session $(p<0.001)$; therefore significant decreases in R-R interval and diastolic time were found.
Left atrial dilatation was also significantly improved after dialysis (before $3.06 \pm 0.5 \mathrm{~mm}$ vs. after $2.73 \pm 0.4 \mathrm{~mm}, p<0.001$ ), with no changes occurred in Ao dimension.

There were no significant differences after HD treatment compared to the baseline value as regards IVSs, IVSd, IVSd by SDS, LVPWD, LVPWD by SDS and IVRT. Systolic function as evaluated by $\mathrm{FS} \%$ and EF\% was not affected by HD, as there were no significant differences between pre- and post-HD findings (FS\% $(p=1)$ and EF\% ( $p=0.699))$. Left ventricular dilatation was the most common abnormal geometric pattern (6 patients had LV dilatation in the baseline val-

Table III. Conventional pulsed wave Doppler and tissue Doppler imaging findings of the patients before and after HD treatment

\begin{tabular}{|c|c|c|c|}
\hline Parameter & Pre-HD $(n=30)$ & Post-HD $(n=30)$ & $P$-value \\
\hline \multicolumn{4}{|c|}{ Conventional echocardiography (CPWD): } \\
\hline $\mathrm{E}[\mathrm{cm} / \mathrm{s}]$ & $125.5 \pm 26.7$ & $91.7 \pm 28.8$ & $<0.001^{*}$ \\
\hline $\mathrm{A}[\mathrm{cm} / \mathrm{s}]$ & $78.8 \pm 21.5$ & $73.6 \pm 26.9$ & 0.415 \\
\hline $\mathrm{E} / \mathrm{A}$ & $1.64 \pm 0.36$ & $1.28 \pm 0.34$ & $<0.001^{*}$ \\
\hline IVRT [ms] & $64.07 \pm 12.86$ & $71.67 \pm 24.58$ & 0.112 \\
\hline $\mathrm{R}-\mathrm{R}[\mathrm{ms}]$ & $639.33 \pm 114.59$ & $563.1 \pm 82.44$ & $0.001^{\star}$ \\
\hline Diastolic time [ms] & $277.5 \pm 67$ & $243.2 \pm 65.09$ & $0.048^{*}$ \\
\hline Deceleration time [ms] & $169.7 \pm 59.16$ & $157.8 \pm 43.51$ & 0.155 \\
\hline \multicolumn{4}{|c|}{ Tissue Doppler imaging (TDI): } \\
\hline \multicolumn{4}{|l|}{ LV lateral wall: } \\
\hline $\mathrm{S}^{\prime}[\mathrm{cm} / \mathrm{s}]$ & $70 \pm 14$ & $73 \pm 21$ & 0.372 \\
\hline $\mathrm{E}^{\prime}[\mathrm{cm} / \mathrm{s}]$ & $130.7 \pm 35.2$ & $109.6 \pm 31.4$ & $0.001^{*}$ \\
\hline$A^{\prime}[\mathrm{cm} / \mathrm{s}]$ & $69 \pm 21$ & $65 \pm 18$ & 0.380 \\
\hline \multicolumn{4}{|l|}{ Septal wall: } \\
\hline $\mathrm{S}^{\prime}[\mathrm{cm} / \mathrm{s}]$ & $70.8 \pm 11.98$ & $65.4 \pm 12.7$ & 0.056 \\
\hline $\mathrm{E}^{\prime}[\mathrm{cm} / \mathrm{s}]$ & $102.3 \pm 22.0$ & $86.7 \pm 26.4$ & $0.004^{*}$ \\
\hline$A^{\prime}[\mathrm{cm} / \mathrm{s}]$ & $70.4 \pm 16.04$ & $68.03 \pm 18.92$ & 0.616 \\
\hline \multicolumn{4}{|l|}{ RV free wall: } \\
\hline $\mathrm{S}^{\prime}[\mathrm{cm} / \mathrm{s}]$ & $115.17 \pm 17.97$ & $112.57 \pm 18.84$ & 0.533 \\
\hline $\mathrm{E}^{\prime}[\mathrm{cm} / \mathrm{s}]$ & $143.77 \pm 22.6$ & $120.17 \pm 33.25$ & $<0.001^{*}$ \\
\hline$A^{\prime}[\mathrm{cm} / \mathrm{s}]$ & $117.97 \pm 35.5$ & $123.0 \pm 39.7$ & 0.450 \\
\hline \multicolumn{4}{|c|}{ Combination of CPWD and TDI: } \\
\hline Septal E/E' & $12.73 \pm 3.44$ & $10.86 \pm 3.24$ & $0.002^{*}$ \\
\hline Lateral E/E' & $10.32 \pm 3.66$ & $8.71 \pm 3.06$ & $0.017^{*}$ \\
\hline Mean E/E' & $11.27 \pm 3.29$ & $9.72 \pm 2.67$ & $0.004^{*}$ \\
\hline
\end{tabular}

Data presented as mean \pm SD. A - late peak diastolic velocity, A' - tissue Doppler late diastolic velocity, $E$ - early peak diastolic velocity, $E^{\prime}$ - tissue Doppler early diastolic velocity, $L V$ - left ventricle, $R V$ - right ventricle, S'-tissue Doppler systolic velocity. ${ }^{*} P$-value was considered significant if $<0.05$. 
ue, which improved to 4 patients after the HD session).

\section{Left ventricular relaxation and compliance}

Table III shows comparisons of CPWD and TDI findings.

The late diastolic velocity (A) did not differ before and after HD while the early diastolic velocities (E) were significantly decreased after HD treatment as compared to the baseline value, as a consequence of these measures, E/A ratios (LV diastolic function) were significantly low after HD treatment. There were no significant differences as regards other mitral inflow velocities including IVRT. Parameters of TDI such as values of the early diastolic velocity E' significantly decreased in post-HD evaluation compared to the pre-HD session in LV wall E' $(p=0.001)$, septal wall E' $(p=0.004)$ and anterior wall E' $(p<0.001)$. The late diastolic mitral velocity $A^{\prime}$ did not significantly differ between pre-and post-HD evaluation (LV A' $(p=0.380)$, septal wall $A^{\prime}(p=0.616)$ and anterior wall $\left.A^{\prime}(p=0.450)\right)$. DTI confirmed the CPWD finding of preserved systolic function after the dialysis session with no significant changes in LV wall S' $(p=0.372)$, septal wall S' $(p=0.056)$ or anterior wall $S^{\prime}(p=0.533)$. Combination of CPWD and TDI revealed that elevated left ventricular filling pressure (lat E/E' > 15) was present in $2(6.6 \%)$ patients in the predialysis value and no one after the dialysis session. This ratio

Table IV. Correlations between pre- and post-HD BNP levels with M-mode echocardiography, Doppler echocardiography and tissue Doppler imaging findings of patients before and after HD treatment $(n=30)$

\begin{tabular}{|c|c|c|c|c|c|}
\hline \multirow[t]{2}{*}{ Parameter } & \multicolumn{2}{|c|}{ Pre-HD BNP } & \multirow[t]{2}{*}{ Parameter } & \multicolumn{2}{|c|}{ Post-HD BNP } \\
\hline & $r$ & $P$-value & & $r$ & $P$-value \\
\hline Mean E/E' & 0.401 & 0.028 & Mean E/E' & 0.38 & 0.038 \\
\hline Heart rate [beats/min] & -0.183 & 0.332 & HR [beats/min] & -0.086 & 0.650 \\
\hline $\mathrm{E}[\mathrm{cm} / \mathrm{s}]$ & 0.016 & 0.934 & $\mathrm{E}[\mathrm{cm} / \mathrm{s}]$ & 0.215 & 0.253 \\
\hline $\mathrm{A}[\mathrm{cm} / \mathrm{s}]$ & 0.249 & 0.185 & $A[\mathrm{~cm} / \mathrm{s}]$ & -0.071 & 0.711 \\
\hline$E / A$ & -0.212 & 0.261 & $E / A$ & 0.285 & 0.127 \\
\hline Diastolic time [ms] & -0.067 & 0.724 & Diastolic time [ms] & -0.203 & 0.282 \\
\hline Deceleration time [ms] & 0.192 & 0.308 & Deceleration time [ms] & -0.179 & 0.344 \\
\hline IVRT [ms] & 0.057 & 0.766 & IVRT [ms] & 0.046 & 0.807 \\
\hline $\mathrm{R}-\mathrm{R}$ [ms] & 0.276 & 0.141 & $\mathrm{R}-\mathrm{R}[\mathrm{ms}]$ & 0.174 & 0.359 \\
\hline Sept E' $[\mathrm{cm} / \mathrm{s}]$ & -0.398 & 0.029 & Sept E' $[\mathrm{cm} / \mathrm{s}]$ & -0.049 & 0.799 \\
\hline Sept E/E' & 0.385 & 0.036 & Sept E/E' & 0.22 & 0.243 \\
\hline Late $E^{\prime}[\mathrm{cm} / \mathrm{s}]$ & -0.452 & 0.012 & Late $E^{\prime}[\mathrm{cm} / \mathrm{s}]$ & -0.270 & 0.037 \\
\hline Late E/E' & 0.291 & 0.025 & Late E/E' & 0.474 & 0.008 \\
\hline Mean E' $[\mathrm{cm} / \mathrm{s}]$ & -0.469 & 0.009 & Mean E' $[\mathrm{cm} / \mathrm{s}]$ & -0.174 & 0.358 \\
\hline LVEDD $[\mathrm{mm}]$ & 0.05 & 0.791 & LVEDD $[\mathrm{mm}]$ & -0.018 & 0.924 \\
\hline LVESD [mm] & 0.028 & 0.883 & LVESD [mm] & 0.032 & 0.866 \\
\hline FS (\%) & -0.126 & 0.506 & FS (\%) & -0.192 & 0.309 \\
\hline EF (\%) & -0.071 & 0.711 & $\mathrm{EF}(\%)$ & -0.191 & 0.311 \\
\hline IVSd [mm] & -0.025 & 0.898 & IVSd [mm] & -0.08 & 0.674 \\
\hline IVSs [mm] & -0.072 & 0.707 & IVSs [mm] & -0.117 & 0.537 \\
\hline LVPWD [mm] & 0.373 & 0.042 & LVPWD [mm] & -0.035 & 0.856 \\
\hline $\mathrm{LA}[\mathrm{mm}]$ & 0.119 & 0.531 & $\mathrm{LA}[\mathrm{mm}]$ & 0.135 & 0.478 \\
\hline Ao $[\mathrm{mm}]$ & -0.216 & 0.251 & Ao $[\mathrm{mm}]$ & 0.068 & 0.72 \\
\hline
\end{tabular}

A - late diastolic velocity, Ao - aorta, E - early diastolic velocity, EF - ejection fraction, FS - fraction shortening, IVRT - isovolumetric relaxation time, IVSd - interventricular septum diastole, IVSS - inter-ventricular septum systole, LA - left atrium, LVEDD - left ventricular end diastolic diameter, LVESD - LV end systolic diameter, LVPWD - posterior wall diameter. P was considered significant if $<0.05$. 
was significantly decreased after the HD session $(p=0.017)$. Elevated mean ventricular filling pressure (mean E/E' > 13) was present in 8 patients before the HD session (26.6\%) and improved after the dialysis session (only 2 patients, $6.6 \%$ ). Also the mean E/E' ratio and septal ventricular compliance E/E' ratio were significantly decreased (improved) after the dialysis session $(p=0.004$ and $p=0.002$, respectively).

\section{Plasma BNP levels}

There was a significant decrease in BNP level after the dialysis session (before HD $374 \pm 412 \mathrm{pg}$ / dl vs. after HD $273 \pm 321 \mathrm{pg} / \mathrm{dl}, p=0.000) .63 .3 \%$ of patients had $>20 \%$ BNP changes after the HD session while $36.7 \%$ had $<20 \%$ BNP changes after the HD session.

Pearson's correlations between BNP levels and different variables are shown in Table IV.

\section{Before HD}

Plasma BNP levels were positively correlated with impaired LV filling pressure as demonstrated by mean $\mathrm{E} / \mathrm{E}$ ' $(r=0.401, p=0.028)$, lateral $E / E "$ $(r=0.291, p=0.025)$ (confirmed by a significant correlation after HD session) and LVPWD ( $r=$ $0.373, p=0.042$ ), but were negatively correlated with septal $\mathrm{E}^{\prime}(r=-0.398, p=0.029)$, lateral wall $\mathrm{E}^{\prime}(r=-0.452, p=0.012)$ and mean E' $(r=-0.469$, $p=0.009)$.

Table V. Correlations of pre-HD and post-mean ventricular filling pressure (E/E') with $M$-mode echocardiography, Doppler echocardiography and tissue Doppler imaging findings of patients before and after HD treatment $(n=30)$

\begin{tabular}{|c|c|c|}
\hline \multirow[t]{2}{*}{ Parameter } & \multicolumn{2}{|c|}{ Pre-HD mean E/E' } \\
\hline & $r$ & $P$-value \\
\hline $\mathrm{E}[\mathrm{cm} / \mathrm{s}]$ & 0.557 & $0.001^{*}$ \\
\hline $\mathrm{A}[\mathrm{cm} / \mathrm{s}]$ & 0.488 & $0.006^{\star}$ \\
\hline Septal E' $[\mathrm{cm} / \mathrm{s}]$ & -0.398 & $0.029^{*}$ \\
\hline Septal E/E' & 0.385 & $0.036^{\star}$ \\
\hline Lateral E' & -0.452 & $0.012^{\star}$ \\
\hline Lateral E/E' & -0.951 & $<0.001^{\star}$ \\
\hline \multirow[t]{2}{*}{ Parameter } & \multicolumn{2}{|c|}{ Post-HD mean E/E' } \\
\hline & $r$ & $P$-value \\
\hline $\mathrm{E}[\mathrm{cm} / \mathrm{s}]$ & 0.526 & $0.003^{*}$ \\
\hline Lateral E/E' & 0.474 & $0.008^{*}$ \\
\hline $\mathrm{LA}[\mathrm{mm}]$ & 0.372 & $0.043^{*}$ \\
\hline
\end{tabular}

\section{After HD}

Plasma BNP concentrations were positively correlated with impaired LV compliance as demonstrated by mean E/E' $(r=0.38, p=0.038)$ and lateral E/E" $(r=0.474, p=0.008)$, but were negatively correlated with decreased lateral wall E' $(r=-0.270, p=0.037)$.

Pre-HD plasma BNP concentrations and pre-HD LVPWD measurement were positively correlated with the duration of dialysis $(r=0.38, p=0.037$; $r=0.41, p=0.024$, respectively).

Correlations between mean E/E' and other echocardiographic parameters are shown in Table V.

\section{Mean E/E' before HD}

Pre-HD mean E/E' was correlated positively with $E$ wave, $A$ wave of CPWD and E/E' of the septal wall while it was correlated negatively with septal E', lateral E' and E/E' of the lateral wall.

\section{Mean E/E' after HD}

Post-HD mean E/E' was correlated positively with $E$ wave of CPWD, LA dimension and finally with $E / E$ ' of the lateral wall.

\section{Discussion}

Left ventricular structure and function abnormalities using CPWD in both adults and children with CKD have been well documented [2-4, 6, 12, 22]. However, data about TDI are very limited in pediatric CKD patients [4, 7, 15]. Children are an ideal group to examine cardiovascular structure and function because they have much lower exposure to 'traditional' risk factors for CVD such as diabetes, smoking, and obesity. The most important result of the present study is that CPWD findings were parallel to TDI parameters in HD children.

Left ventricular hypertrophy is usually associated with reduced stress on the LV wall and increased SV in the early stages of systemic hypertension [23]. In the present study, children with ESRD exhibited an elevated thickness of the IVS and LVPW (positive correlation with the duration of dialysis) and showed a trend to increased (as detected by increased LVEDD before HD) LVEDD or signs of $\mathrm{LVH}$. These findings are compared to ESRD adult patients' findings [23, 24]. In the present study, LA dilatation is one of the common geometric abnormal patterns reflecting diastolic dysfunction. This dilatation also significantly improved after dialysis in our study. During ventricular diastole the LA is directly exposed to LV filling pressure, so increased LA volume and size may be a reflection of the duration and severity of diastolic dysfunction [25]. The effect of HD on LA dimension could be explained by the factors 
that affect LV filling. LA pressure increases leading to augmented LA dimensions either due to preload increases and/or LV compliance decreases. In regular HD patients, the LA parameters are reported as markers of chronic diastolic dysfunction. However, some investigators showed that LA parameters were similar in the control group [26, 27]. Myocardial velocity by TDI and LA parameters have been considered relatively preload independent measurements of diastolic function and can reflect $\mathrm{LV}$ diastolic dysfunction accurately $[25,28]$.

This study has studied the effects of hemodialysis-mediated preload reduction on the LV diastolic function. The LV diastolic function was calculated using both flow Doppler and TDI in patients with ESRD before and immediately after HD. HD resulted in a decrease in body water content. This decrease was accompanied by a decrease in intravascular volume, which leads to a decrease in the dimensions of the LV, as we have demonstrated in our study. Heart rate was significantly higher after the HD session; therefore significant decreases in R-R interval and diastolic time were found. This may be explained by the inflammatory tissue reaction which occurred after dialysis, or be due to hyperdynamic circulation as a compensation mechanism for decreased blood pressure or dehydration.

In the present study, the finding of mitral annular diastolic velocities, derived by CPWD, showed that the early diastolic velocities (E) were significantly improved after dialysis, whereas there was no significant difference with respect to peak late diastolic velocities (A) between pre- and post-HD measurements. As a result of these measures, E/A ratio was significantly lower after the HD session. On the other hand, as in our patients, the initial abnormality of cardiac function is usually diastolic dysfunction; these results are comparable to other studies using HD as a clinical model of preload reduction [29-31]. Several factors can influence E/A ratio, including LA pressure and preload [32].

The TDI is a relatively new method used to evaluate the diastolic function of LV, assuming TDI indices, in contrast to CPWD, may be less preload dependent and provide a more accurate measure of diastolic function. Moreover, some studies have shown that the effect of preload can be corrected by the index of LV filling pressure: the combination of cPWD and TDI $[16,33]$. Decreased passive left ventricular filling by TDI is reflected by decreased trans-mitral velocity (E'), which can be partially compensated only by increased LA contraction. In our study, the index of LV filling pressure (E/E'), which is a better indicator of diastolic function using both CPWD and TDI, showed considerable differences between pre- and post-HD measurements.
The use of TDI at the mitral annulus allows the investigator to estimate the filling pressure. An E/E' ratio above 12 is indicative of diastolic dysfunction (possible diastolic dysfunction when the ratio is between 8 and 12) [34], and it was significantly associated with various variables (in the present study, pre-dialysis mean E/E' correlated positively with pre-BNP, while post-HD mean E/E' correlated positively only with post-BNP, $E$ and LA dimension) in HD patients. This indicates that BNP was a good predictor of early diastolic dysfunction measured by E/E'. However, we found that age and $E / E$ ' in correlation analysis are not associated, which was concordant with other pediatric CKD studies [33].

In our study, the difference between TDI measurements before and after HD sessions means that it is relatively preload dependent. The effects of changes in preload on LV diastolic function obtained via TDI echocardiography were reported in some studies; they reported that diastolic velocities assessed by TDI are relatively preload dependent $[35,36]$. There was a reported relationship between E/E' and left ventricular mass index (LVMI) in pediatric dialysis studies $[8,33,37]$. They postulated that E/E' ratio can be used as an index of LV filling pressure and consequently to estimate LV myocardial compliance at end diastole, especially in patients with preserved systolic function. The reduced LV compliance due to LVH results in LV diastolic dysfunction, with reduced passive LV filling as a consequence.

In contrast to adults, LV systolic function usually remains normal in our patients compared to $\mathrm{LVH}$ in children on dialysis. EF and FS values in our study are similar to previous pediatric studies [4, 5, 12, 38]. However, Mitsnefes et al. [38] demonstrated diminished contractile reserve during exercise in children on dialysis. It is possible that more subtle changes in LV function are unmasked due to evaluation during exercise. However, there was an insignificantly lower S' measurement (systolic function) in our patients (both pre- and post-HD) in the septal wall of the LV, indicating an initial systolic dysfunction in ESRD patients, and this finding indicated high sensitivity of TDI in detecting early systolic dysfunction. Speckle-tracking echocardiography may reveal early myocardial dysfunction in the absence of systolic dysfunction measured by conventional ultrasound or TDI in children with ESRD [16].

Civilibal et al. [39] clearly showed that CPWD findings parallel TD findings in pediatric dialysis patients. There were significant correlations between the indices of CPWD (A, E and A/E ratio) and TDI (A', E'\& E'/A'). Therefore, they considered that CPWD is a good method to evaluate LV diastolic function in patients on HD. However, these results are questionable due to the small sample size. 
In spite of the productive recording of BNP information in adults with CKD, reports about this hormone in children are scarce [20]. A few research studies have detected the clinical usefulness of BNP in congenital heart disease in children [4043], congestive heart failure (CHF) [44] and normal renal function. Similarly, Koch, et al. [40] and Law et al. [41] reported a variable range of BNP concentrations in patients with CVD, but significant elevation in BNP was restricted to patients with systolic or diastolic ventricular dysfunction. It is better to evaluate the possible application of this biomarker in CKD children as it is a widely known parameter for occurrence of CVD.

The normal range of BNP concentration in newborns and children with normal kidney function has been reported $[42,43]$. Koch and Singer [44] reported in healthy children BNP levels less than $32.7 \mathrm{pg} / \mathrm{ml}$ (at the age from 2 weeks to 17.6 years). Mean plasma BNP concentrations in children from the age of 2 weeks are characteristically lower than in adults [44]. Plasma BNP levels are nearly the same between boys and girls in children $<10$ years of age [44] but significant differences were found between boys and girls after puberty [45]. Thereafter, BNP concentration rises significantly with age, during adolescence and adulthood, [45]. Therefore, a 10-year increase in age is accompanied by a 1.4-fold elevation in plasma BNP in adults [43]. Accordingly, these normal reference values will help to avoid CV condition misclassification in children with the biomarker NT-proBNP. In patients with or without deteriorating renal function, the pediatric optimum cut-off values for diagnosing cardiovascular disorders, especially with LV diastolic and systolic dysfunction, are different from adult values.

In this study, plasma BNP levels (pre- vs. postHD) decreased significantly after high-flux HD and the BNP changes decreased by $<20 \%$ in $36.7 \%$ of patients and $>20 \%$ in $63.3 \%$ of patients probably because of the unloading of the heart and the removal of the peptide during HD. Earlier, Wahl et al. [46] reported that natriuretic peptides such as NT-proBNP are completely removed during HD because they measured this peptide in the dialysate. A recent study performed by Laveborn et al. [47] also detected a significant decrease (pre- vs. post-HD) in NT-proBNP and TnT by using highflux membranes, but cardiac biomarkers increase slightly by using low-flux membranes. Because of the significant lowering of the post-DH plasma peptides and the equality of sieving coefficients of the dialyzers used, it could be supposed that the main cause of the results in this study is the convective effect and not a substantial amelioration in cardiac function. Plasma NT-proBNP and BNP demonstrate the hemodynamic myocardial stress distinct from the underlying pathology. Most HD patients have elevated ventricular wall stress because of the fluid volume and pressure overload on the cardiac muscle, and their severity and prognosis can be detected by NT-proBNP and BNP levels. The cardiovascular stress was not significantly lowered by the convective therapy, as reflected by the pre-HD vasoactive peptide levels in this study [46]. The BNP may be elevated in volume-overloaded patients independently of cardiac dysfunction as in those with nephritic syndrome, which is seen in animals experimentally and in children with acute post-streptococcal glomerulonephritis (NT-proBNP) $[8,47]$.

In this study, we detected a positive correlation between pre/post-HD-BNP levels measured and different echocardiographic variables which mirror left ventricular diastolic function before and after HD. In contrast, Ito et al. [48] did not report any correlation of pre- or post-HD BNP levels with any TDI variables when measuring the transmitral inflow. Some researchers [48-50] have reported that serum NT-pro BNP levels were strongly correlated with the parameters of LV dysfunction. These researchers summarized that BNP may predict an abnormal cardiac geometry in CKD. This finding is in accordance with a report in the literature about BNP in HD adults [20]. In children with normal renal function, there was a significant positive correlation between BNP level and CHF grades and a cut-off value was detected for each grade by using a receiver operating characteristics (ROC) curve to predict grades of CHF based on blood BNP level [49].

Our study has some limitations, including the fact that no invasive studies were done to correlate hemodynamic measurements with TDI measurements as the study was done on pediatric patients, and no invasive measurements were made to directly measure the preload changes. This was a single-center, cross-sectional study that included a limited number of patients. Although hemodialysis is frequently used in studies of preload effects on certain parameters, factors other than preload such as afterload reduction (lower diastolic pressure) and reduction in acidemia might be confounding the results.

In conclusion, the indices of diastolic function obtained by CPWD are preload dependent and the loading status of the patient should be taken into account during the application of these indices to the evaluation of myocardial performance. The TDI findings correlate well with conventional echocardiography. We still suggest that CPWD is a good method to evaluate LV diastolic function in pediatric dialysis patients. However, the indices obtained by TDI may be adjunctive parameters to cPWD for detecting myocardial dysfunction, such 
as $\mathrm{LV}$ relaxation and filling pressure indices. One should be careful when interpreting BNP concentration as ESRD children may display high values of BNP independent of cardiac dysfunction in volume-overloaded patients. Thus, the diagnostic usefulness of BNP in the occurrence of renal dysfunction may be compromised to a certain extent.

\section{Acknowledgments}

This study was funded by the National Research Centre (grant number 10010008).

\section{Conflict of interest}

The authors declare no conflict of interest.

\section{References}

1. US Renal Data System. 1998 annual report. The National Institute of Diabetes and Digestive and Kidney Disease. Am J Kidney Dis 1998; 32 (Suppl 1): S81-8.

2. Al-Biltagi M, Tolba OA, ElHafez MA, Abo-Elezz AA, El Kady EK, Hazza SM. Oxidative stress and cardiac dysfunction in children with chronic renal failure on regular hemodialysis. Pediatr Nephrol 2016; 31: 1329-39.

3. Goicoechea M, Garcia de Vinuesa S, Gomez-Campdera F, Luno J. Predictive cardiovascular risk factors in patients with chronic kidney disease. Kidney Int 2005; 67 (Suppl 93): S35-8.

4. Saygılı A, Yıldırım SV, Cengiz N, Uslu Y, Tokel K, Saatci U. Assessment of left ventricular diastolic function by Doppler tissue imaging in children with end-stage renal disease. Acta Paediatr 2005; 94: 1055-9.

5. Mitsnefes MM. Cardiovascular complications of pediatric chronic kidney disease. Pediatr Nephrol 2008; 23: 27-9.

6. Ucar T, Tutar E, Yalcinkaya F, et al. Global left-ventricular function by tissue Doppler imaging in pediatric dialysis patients. Pediatr Nephrol 2008; 23: 779-85.

7. Foley RN, Parfrey PS, Sarnak MJ. Epidemiology of cardiovascular disease in chronic renal disease. J Am Soc Nephrol 1998; 9 (12 Suppl): S16-23.

8. Yeh CF, Huang HS, Chang SN, et al. Biomarkers and echocardiography for evaluating the improvement of the ventricular diastolic function after surgical relief of hydronephrosis. PLoS One 2017, DOI: https://doi. org/10.1371/journal.pone.0188597.

9. Levin A, Singer J, Thompson CR, Ross H, Lewis M. Prevalent left ventricular hypertrophy in the predialysis population: identifying opportunities for intervention. Am J Kidney Dis 1996; 27: 347-54.

10. London GM. Cardiovascular disease in chronic renal failure: pathophysiologic aspects. Semin Dial 2003; 16: 85-94.

11. London GM, Parfrey PS. Cardiac disease in chronic uremia: pathogenesis. Adv Ren Replace Ther 1997; 4: 194-201.

12. Elshamaa MF, Sharaf EA, Farid YA, Elghoroury EA. Plasma nitric oxide level in myocardial disorders with left ventricular diastolic dysfunction. J Clin Basic Cardiol 2006; 9: 23-6.

13. Derumeaux G, Douillet R, Troniou A, et al. Distinguishing between physiologic hypertrophy in athletes and primary hypertropic cardiomyopathies. Importance of tissue color Doppler. Arch Mal Coeur Vaiss 1999; 92: 201-10.
14. Ommen SR, Nishimura RA, Appleton CP, Miller FA, Oh JK, Redfield MM. Clinical utility of Doppler echocardiography and tissue Doppler imaging in the estimation of left ventricular filling pressures: a comparative simultaneous Doppler-catheterization study. Circulation 2000; 102: 1788-94.

15. Edvardsen T, Gerber B, Garot J, Bluemke D, Lima J, Smiseth OA. Quantitative assessment of intrinsic regional myocardial deformation by Doppler strain rate echocardiography in humans: validation against three-dimensional tagged magnetic resonance imaging. Circulation 2002; 106: 50-6.

16. van Huis M, Schoenmaker NJ, Groothoff JW, et al. Impaired longitudinal deformation measured by speckle-tracking echocardiography in children with endstage renal disease. Pediatr Nephrol 2016; 31: 1499-508.

17. Derumeaux G, Mulder P, Richard V, Chagraoui A, Nafeh C, Bauer F. Tissue Doppler imaging differentiates physiological from pathological pressure-overload left ventricular hypertrophy in rats. Circulation 2002; 105: 1602-8.

18. Rodriguez L, Garcia M, Ares M, Griffin BP, Nakatani S, Thomas JD. Assessment of mitral annular dynamics during diastole by Doppler tissue imaging: comparison with mitral Doppler inflow in subjects without heart disease and in patients with left ventricular hypertrophy. Am Heart J 1996; 131: 982-7.

19. Hayashi SY, Brodin LA, Alvestrand A, Lind B, Stenvinkel P, Mazza do Nascimento M. Improvement of cardiac function after haemodialysis. Quantitative evaluation by colour tissue velocity imaging. Nephrol Dial Transplant 2004; 19: 1497-506.

20. Quali S, Bougmiza I, Abroug S, et al. Relationship of brain natriuretic peptide concentrations to left ventricular function and adverse outcomes in children with endstage renal disease undergoing hemodialysis. Pediatr Cardiol 2011; 32: 568-77.

21. Schiller NB, Shah PM, Crawford M, et al. Recommendations for quantitation of the left ventricle by two-dimensional echocardiography. American Society of Echocardiography Committee on Standards, Subcommittee on Quantitation of Two-Dimensional Echocardiograms. J Am Soc Echocardiogr 1989; 2: 358-67.

22. Civilibal M, Caliskan S, Oflaz H, et al. Traditional and 'new' cardiovascular risk markers and factors in pediatric dialysis patients. Pediatr Nephrol 2007; 22: 1021-9.

23. Raine AEG, Schwarz U, Ritz E. Hypertension and cardiac problems. In: Oxford Textbook of Clinical Nephrology. $2^{\text {nd }}$ ed. Davison AM, Cameron JS, Grunfeld JP, Kerr DNS, Ritz E, Winearls CG (eds). Oxford University Press, Oxford 1998; 1885-918.

24. London GM, Marchais SJ, Guérin AP, Fabiani F, Métivier $F$. Cardiovascular function in hemodialysis patients. In: Advances in Nephrology. Vol. 20. Grünfeld JP, Bach JF, Funck-Brentano JL, Maxwell MH (eds). Mosby Year Book Publishers, St. Louis, 1991; 249-73.

25. Fijalkowski M, Koprowski A, Gruchala M, et al. Effect of preload reduction by hemodialysis on myocardial ultrasonic characterization, left atrial volume, and Doppler tissue imaging in patients with end-stage renal disease. J Am Soc Echocardiogr 2006; 19: 1359-64.

26. Appleton CP, Galloway JM, Gonzalez MS, Gaballa M, Basnight MA. Estimation of left ventricular filling pressures using two-dimensional and Doppler echocardiography in adult patients with cardiac disease. Additional value of analyzing left atrial size, left atrial ejection fraction and the difference in duration of pulmonary venous and 
mitral flow velocity at atrial contraction. J Am Coll Cardiol 1993; 22: 1972-82.

27. Barberato SH, Mantilla DE, Misocami MA, et al. Effect of preload reduction by hemodialysis on left atrial volume and echocardiographic Doppler parameters in patients with endstage renal disease. Am J Cardiol 2004; 94: 1208-10.

28. le EHY, Vletter WB, Ten Cate FJ, et al. Preload dependence of new Doppler techniques limits their utility for left ventricular diastolic function assessment in hemodialysis patients. J Am Soc Nephrol 2003; 14: 1858-62.

29. Gerede DM, Turhan S, Kaya CT, et al. Effects of hemodialysis on Tei Index: comparison between flow Doppler and tissue Doppler imaging. Echocardiography 2015; 32: 1520-6.

30. Agmon Y, Oh JK, McCarthy JT, et al. Effect of volume reduction on mitral annular diastolic velocities in hemodialysis patients. Am J Cardiol 2000; 85: 665-8, A11.

31. Barberato SH, Mantilla DE, Misocami MA, et al. Effect of preload reduction by hemodialysis on left atrial volume and echocardiographic Doppler parameters in patients with end-stage renal disease. Am J Cardiol 2004; 94: 1208-10.

32. Koga S, Ikeda S, Matsunaga K, et al. Influence of hemodialysis on echocardiographic Doppler indices of the left ventricle: changes in parameters of systolic and diastolic function and Tei index. Clin Nephrol 2003; 59: 180-5.

33. Mitsnefes MM, Kimball TR, Border WL, et al. Impaired left ventricular diastolic function in children with chronic renal failure. Kidney Int 2004; 65: 1461-6.

34. Arques S, Roux E, Luccioni R. Current clinical applications of spectral tissue Doppler echocardiography (E/E' ratio) as a noninvasive surrogate for left ventricular diastolic pressures in the diagnosis of heart failure with preserved left ventricular systolic function. Cardiovasc Ultrasound 2007; 5: 16.

35. Oguzhan A, Arinc H, Abaci A, Topsakal R, Eryol NK, Ozdogru I. Preload dependence of Doppler tissue imaging derived indexes of left ventricular diastolic function. Echocardiography 2005; 22: 320-5.

36. Drighil A, Madias JE, Mathewson JW, El Mosalami H, E Badaoui N, Ramdani B. Hemodialysis: effects of acute decrease in preload on tissue Doppler imaging indices of systolic and diastolic function of the left and right ventricles. Eur J Echocardiogr 2008; 9: 530-5.

37. van Huis M, Schoenmaker NJ, Groothoff JW, et al. Impaired longitudinal deformation measured by speckle-tracking echocardiography in children with end-stage renal disease. Pediatr Nephrol 2016; 31: 1499-508.

38. Mitsnefes MM, Kimball TR, Witt SA, Glascock BJ, Khoury PR, Daniels SR. Left ventricular mass and systolic performance in pediatric patients with chronic renal failure. Circulation 2003; 107: 864-8.

39. Civilibal M, Caliskan S, Oflaz $\mathrm{H}$, et al. Left ventricular function by 'conventional' and 'tissue Doppler' echocardiography in pediatric dialysis patients. Nephrology 2009; 14: 636-42

40. Koch A, Zink S, Singer H. B-type natriuretic peptide in paediatric patients with congenital heart disease. Eur Heart J 2006; 27: 861-6.

41. Law YM, Keller BB, Feingold BM, Boyle GJ. Usefulness of plasma B-type natriuretic peptide to identify ventricular dysfunction in pediatric and adult patients with congenital heart disease. Am J Cardiol 2005; 95: 474-8.

42. Law YM, Hoyer AW, Reller MD, Silberbach M. Accuracy of plasma B-type natriuretic peptide to diagnose significant cardiovascular disease in children: the Better
Not Pout Children! Study. J Am Coll Cardiol 2009; 54 1467-75.

43. Westerlind A, Wahlander $\mathrm{H}$, Lindstedt $\mathrm{G}$, Lumberg PA Holmgren D. Clinical signs of heart failure are associated with increased levels of natriuretic peptides types $B$ and $A$ in children with congenital heart defects or cardiomyopathy. Acta Paediatr 2004; 93: 340-5.

44. Koch A, Singer $H$. Normal values of B-type natriuretic peptide in infants, children, and adolescents. Heart 2003; 89: 875-8.

45. Uhlin F, Odar-Cederlöf I, Theodorsson E, Fernström A Vasoactive peptide levels after change of dialysis mode. Nephron Extra 2015; 5: 67-78.

46. Wahl HG, Graf S, Renz H, Fassbinder W. Elimination of the cardiac natriuretic peptides B-type natriuretic peptide (BNP) and N-terminal proBNP by hemodialysis. Clin Chem 2004; 50: 1071-4.

47. Laveborn E, Lindmark K, Skagerlind M, Stegmayr B. NT-proBNP and troponin T levels differ after haemodialysis with a low versus high flux membrane. Int J Artif Organs 2015; 38: 69-75.

48. Untersteller K, Girerd N, Duarte K, et al. NT-proBNP and echocardiographic parameters for prediction of cardiovascular outcomes in patients with CKD stages G2-G4. Clin J Am Soc Nephrol 2016; 11: 1978-88.

49. Zoair AM, Abdel-Hafez MA, Mawlana W, Sweylam MA. Serum levels of $\mathrm{N}$-terminal-pro B-type natriuretic peptide as a diagnostic marker for left ventricular dysfunction in children with end-stage renal disease on hemodialysis. Saudi J Kidney Dis Transpl 2016; 27: 1114-22.

50. Noori NM, Teimouri A, Anvari N. Diagnostic value of $N$ terminal pro B type natriuretic peptide (NT-pro BNP) in cardiac involvement in patients with betathalassemia. Int J Pediatr 2017; 5: 4641-62. 